\title{
Transient Abnormal Myelopoiesis and AML in Down Syndrome: an Update
}

\author{
Neha Bhatnagar $^{1} \cdot$ Laure Nizery $^{2} \cdot$ Oliver Tunstall $^{3} \cdot$ Paresh Vyas $^{4} \cdot$ Irene Roberts $^{5}$
}

Published online: 10 August 2016

(C) The Author(s) 2016. This article is published with open access at Springerlink.com

\begin{abstract}
Children with constitutional trisomy 21 (Down syndrome (DS)) have a unique predisposition to develop myeloid leukaemia of Down syndrome (ML-DS). This disorder is preceded by a transient neonatal preleukaemic syndrome, transient abnormal myelopoiesis (TAM). TAM and ML-DS are caused by co-operation between trisomy 21 , which itself perturbs fetal haematopoiesis and acquired mutations in the key haematopoietic transcription factor gene GATA1. These mutations are found in almost one third of DS neonates and are frequently clinically and haematologcially 'silent'. While the majority of cases of TAM undergo spontaneous remission, $\sim 10 \%$ will progress to ML-DS by acquiring transforming mutations in additional oncogenes. Recent advances in the unique biological, cytogenetic and molecular characteristics of TAM and ML-DS are reviewed here.
\end{abstract}

Keywords Transient abnormal myelopoiesis · Down syndrome $\cdot$ Acute leukaemia $\cdot$ Myeloproliferative disorders

This article is part of the Topical Collection on Myeloproliferative Disorders

Irene Roberts

irene.roberts@paediatrics.ox.ac.uk

Neha Bhatnagar

neha.bhatnagar@ouh.nhs.uk

Laure Nizery

laure.nizery@gmail.com

Oliver Tunstall

oliver.tunstall@uhbristol.nhs.uk

Paresh Vyas

paresh.vyas@imm.ox.ac.uk

\section{Introduction}

Population studies show that children with Down syndrome due to constitutional trisomy 21 have a markedly increased risk of developing acute leukaemia compared with children without Down syndrome [1]. Both myeloid leukaemia, known as myeloid leukaemia of Down syndrome (ML-DS), and acute lymphoblastic leukaemia are increased by 150 - and $~ 30$-fold, respectively $[1,2]$. ML-DS has a distinct natural history and clinical and biological features (reviewed in [3, 4]). It virtually always develops before the age of 5 years, and the acute leukaemia is preceded by a clonal neonatal preleukaemic syndrome known as transient abnormal myelopoiesis (TAM) that is unique to Down syndrome [3, 4].

TAM is characterised by increased circulating blast cells that harbour acquired N-terminal truncating mutations in the key haematopoietic transcription factor gene GATA1 [5-10]. Around $10-15 \%$ of neonates with Down syndrome have a diagnosis of TAM with blasts $>10 \%$ and typical clinical fea-
1 Children's Hospital, John Radcliffe Hospital, Oxford University Hospitals NHS Foundation Trust, Oxford OX3 9DU, UK

2 Paediatric Intensive Care Unit, Robert Debré Hospital, 48 Boulevard Sérurier, 75019 Paris, France

3 Bristol Royal Hospital for Children, Paul O'Gorman Building, Upper Maudlin St, Bristol BS2 8B, UK

4 Molecular Haematology Unit, Weatherall Institute of Molecular Medicine, University of Oxford, John Radcliffe Hospital, Oxford OX3 9DS, UK

5 Department of Paediatrics, Children's Hospital, University of Oxford, John Radcliffe Hospital, OX3 9DU Oxford, UK 
tures that require close monitoring in the neonatal period since the mortality rate may be up to $20 \%$. A further $10-15 \%$ of neonates with Down syndrome have one or more acquired GATA1 mutations in association with a low number of circulating blast cells $(<10 \%)$ and have clinically and haematologically silent disease (silent TAM) [11••]. In the majority of cases of TAM and silent TAM, the GATA1 mutant clone goes into complete and permanent remission without the need for chemotherapy. However, $10-20 \%$ of neonates with TAM and silent TAM subsequently develop ML-DS in the first 5 years of life when persistent GATA1 mutant cells acquire additional oncogenic mutations, most often in cohesin or epigenetic regulator genes $[12 \bullet \bullet, 13]$. This review article discusses the recent clinical and biological advances in TAM and ML-DS and how these may impact on clinical management.

\section{Cellular and Molecular Pathogenesis of TAM and ML-DS}

The cellular and molecular events involved in initiation and evolution of TAM and ML-DS can best be understood as a three-step model which requires the presence within a fetal liver-derived haematopoietic stem or progenitor cell of (i) trisomy 21, (ii) an acquired GATA1 mutation, and (iii) at least one additional oncogenic mutation (Fig. 1).

\section{(i) Perturbation of fetal haematopoiesis by trisomy 21}

The initial event in trisomy 21 -associated preleukaemic and leukaemic conditions is the perturbation of fetal haematopoiesis by trisomy 21 itself. It is known that by late in the first trimester of fetal life, haematopoiesis in the liver is abnormal in fetuses with trisomy 21 and that these changes precede the acquisition of GATA1 mutations $[14,15 \bullet \cdot$. Specifically, trisomy 21 causes an increase in the numbers of megakaryocyte-erythroid progenitors (MEP) and an increase in the size and characteristics of the immunophenotypic haematopoietic stem cell (HSC) compartment $\left[15 \bullet^{\circ}\right]$. HSC and multipotent myeloid progenitors in trisomy 21 fetal liver proliferate more in vitro compared with normal fetal liver at the same stage of development and have increased erythroid-megakaryocyte output and gene expression [15०•]. Despite the increase in megakaryocytes (MK) in trisomy 21 fetal liver, MK differentiation is impaired and platelet counts are reduced both in fetal blood and in neonates with Down syndrome suggesting that trisomy 21 itself causes dysmegakaryopoiesis $[11 \bullet \bullet, 14,15 \bullet \bullet]$.

The molecular basis for these dramatic changes in fetal erythro-megakaryopoiesis is not yet clear. Some, but not all, of the features can be recapitulated either in elegant animal models [16-20] or in studies in human embryonic stem cells (ESC) and induced pluripotent stem cells (iPSC) $[21,22]$. Together, these studies have implicated increased expression of various genes on chromosome 21 , in particular $E R G$ and DYRKIa, as important mediators of the abnormal megakaryopoiesis, although this does not seem to be sufficient to cause leukaemia in trisomic or disomic mouse models even when co-expressed with an N-terminally truncated GATA1 gene. Interestingly, recent data using a panel of iPSC lines, suggest that trisomy of $R U N X 1, E T S 2$, and $E R G$ might be sufficient, in combination with mutant GATA1, to explain many of the haematopoietic abnormalities seen in primary human fetal liver and TAM cells. Despite these interesting findings, it is now clear that trisomy 21 causes genome-wide changes in gene expression directly or indirectly affecting multiple genes on most chromosomes [23].

(ii) N-terminal truncating GATA1 mutations in TAM and ML-DS

The link between acquired mutations in the GATA1 gene and ML-DS was first identified more than 12 years ago in John Crispino's lab [5] and rapidly followed by studies from a number of groups confirming the link with trisomy 21 as well as showing the same N-terminal truncating mutations in TAM [6-10]. The GATA1 mutations that can be detected in all cases disappear when TAM (or ML-DS) enter remission indicating that these are acquired events $[9,12 \bullet \cdot$. Application of highly sensitive next-generation sequencing (NGS)-based methodology has recently shown that GATA1 mutations are present in all cases of TAM or ML-DS and that they are present in $25-30 \%$ of all neonates with Down syndrome [11••]. This means that GATA1 mutations are necessary for the development of TAM/ML-DS, that they are acquired prior to birth in fetal cells and that they occur at an astonishingly high frequency. It seems likely that acquisition of such mutations confers a selective growth advantage to these cells during fetal life. The presence of multiple GATA1 mutant clones in up to $25 \%$ of neonates with Down syndrome is consistent with this $[9,11 \bullet \bullet]$; however, the reason for their high frequency in Down syndrome remains unknown (these mutations are not found in normal, disomic cord blood) $[9,11 \bullet \bullet]$.

The vast majority of acquired GATA1 mutations $(\sim 97 \%)$ are found in exon 2 and the remainder in exon 3.1 of the GATA1 gene, including insertions, deletions and point mutations [10]. These mutations lead to expression of a truncated GATA1s protein $[5,6]$ and the type of GATA1 mutation does not predict which patients with TAM will later progress to ML-DS [10]. Since the GATA1 gene is on the $\mathrm{X}$ chromosome, haematopoietic cells harbouring GATA1 mutations express only GATA1s and no longer have the ability to produce the full-length GATA1 protein [5]. The main physiological role of GATA1 is as a regulator of normal megakaryocyte and erythroid differentiation [24]. How GATA1 transforms trisomy 21 fetal haematopoietic cells is unclear. Forced 
Natural history and pathogenesis of TAM and ML-DS

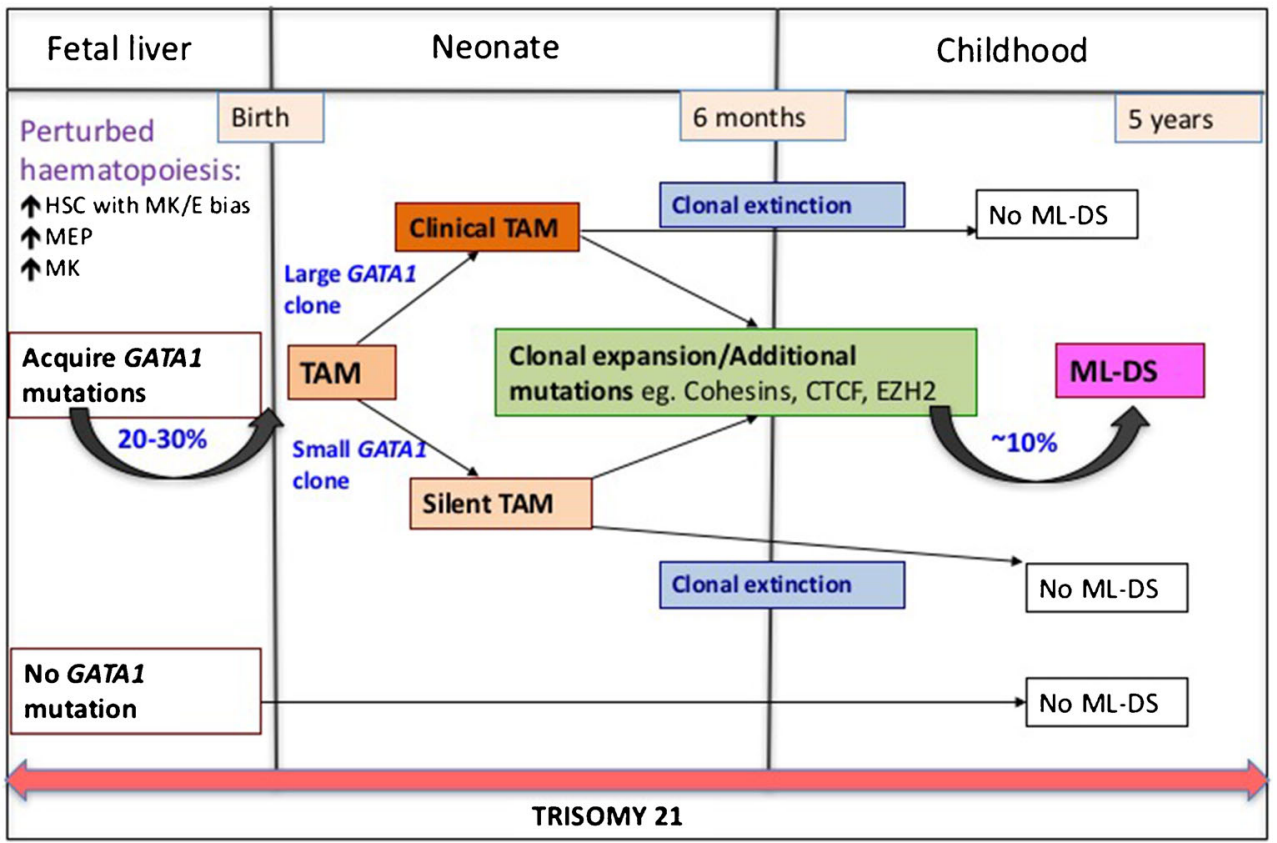

Fig. 1 Natural history and pathogenesis of TAM and ML-DS. Schematic representation of molecular, biological and clinical data, indicating that transient abnormal myelopoiesis (TAM) and myeloid leukaemia of Down syndrome $(M L-D S)$ are initiated before birth when fetal liver haematopoietic stem and progenitor cells $(H S P C)$ trisomic for chromosome 21 demonstrate perturbed haematopoiesis with an expansion of megakaryocyte-erythroid progenitors $(M E P)$ and megakaryocytes. These cells subsequently acquire N-terminal truncating GATA1 mutations resulting in TAM in late fetal or early neonatal life. Although most cases of TAM spontaneously and permanently remit ( $\sim 90 \%)$ by the age of 6 months, in $\sim 10 \%$ of cases, additional genetic/epigenetic events lead to further clonal expansion resulting in ML-DS before the age of 5 years expression of GATA1s in fetal liver haematopoietic progenitors from GATA1 wild-type mice causes marked expansion of megakaryoblastic progenitors, supporting a gain of function mechanism $[25,26]$ and interestingly, Banno et al. using a trisomy 21 iPSC model also found that an increased level of expression of GATA1s might be responsible for the aberrant megakaryopoiesis they observed [27]. However, whether this is the main mechanism in trisomy 21 human cells and how GATA1s might transform trisomy 21 fetal haematopoietic cells remains an interesting question.

(iii) Mutational landscape of ML-DS

The presence of an N-terminal truncating mutation in GATA1 is necessary, but insufficient, for development of ML-DS. Recent whole genome and whole exome sequencing studies of ML-DS provide insight into the additional genetic events which co-operate with GATA1 mutations and trisomy 21 to further transform haematopoietic cells from a usually transient preleukaemic syndrome (TAM) to an acute leukaemia (ML-DS), which is inexorably fatal unless eradicated with chemotherapy [12••, 13]. These show a high frequency of mutations $(\sim 50 \%)$ in all the key cohesin component genes (RAD21, STAG2, SMC3 and $S M C 1 A)$, as well as in CTCF $(\sim 20 \%)$ and in epigenetic regulators such as EZH2 and KANSL1 $(45 \%)$ [12••]. These genes encode proteins important for transcription regulation and long-range interactions that may be particularly vulnerable to disruption in trisomic cells. A smaller proportion of patients had mutation in RAS pathway genes (NRAS, KRAS, $C B L, P T P N 11$ and NF1) $[12 \bullet \bullet, 13]$ that are also seen at high frequency in other childhood leukaemias, such as juvenile myelomonocytic leukaemia [28].

(iv) Role of the haematopoietic microenvironment

Although the data from primary human tissues as well as ESC and iPSC, indicate that trisomy 21 causes cell intrinsic changes in fetal haematopoietic stem and progenitor cells, the fetal liver haematopoietic microenvironment may also contribute both to these changes and to expansion and/or maintenance of the mutant GATA1 clone in TAM. Indeed, the natural history and clinical features of TAM clearly show this to be a fetal liver disease (see below). The nature of the growth factors that might mediate abnormal fetal haematopoiesis in TAM is unclear, although differences in the expression or responsiveness to the developmentally regulated IGF signalling pathway remain an attractive candidate [29]. 


\section{TAM: Clinical Features}

TAM has a very variable clinical presentation: at one end of the spectrum, it may be detected as an incidental finding on review of a blood film in an otherwise well baby (10-25\% neonates) and at the other end of the spectrum, neonates with TAM may be very sick with disseminated leukaemic infiltration (10-20\% of neonates) presenting with massive hepatosplenomegaly, effusions, co-agulopathy and multiorgan failure $[30-32,33 \bullet \cdot]$. The majority of neonates with clinical TAM (i.e. blasts $>10 \%$ ) will have one or more of the well recognised clinical features of TAM which are summarised in Table 1. Amongst these features, hepatomegaly, splenomegaly, pericardial/pleural effusions and skin rash are seen more frequently in neonates with TAM compared with neonates without any GATA1 mutations. Jaundice, on the other hand, is common in neonates with Down syndrome with or without TAM [11••, 30-32]. Importantly, however, as no single clinical feature is specific for TAM, it is essential to review the blood film of all neonates with Down syndrome to avoid missing cases of TAM and to assess the significance of the clinical features shown in Table 1 [11••]. This is also important in the setting of delayed onset or prolonged hyperbilirubinaemia in neonates with Down syndrome as this may be the presenting feature of progressive TAM-associated liver fibrosis that may be fatal. Although the majority of cases of TAM present within the first few days of life, TAM may also present in fetal life either with hydrops fetalis or with features similar to those presenting postnatally [34•].

\section{TAM: Laboratory Features}

TAM causes several haematological abnormalities. Characteristically, the main features are leucocytosis and increased peripheral blood blasts. Leucocytosis is present in 30$50 \%$ of cases of TAM and typically includes increased neutrophils, myelocytes, monocytes and basophils $[11 \bullet \bullet, 31,33 \bullet \bullet$. The platelet count may be elevated, normal or reduced, and thrombocytopenia is not more common in neonates with TAM than in DS neonates without TAM [11••]. Similarly, although the median haemoglobin is lower in neonates with TAM compared with neonates with Down syndrome without TAM, anaemia is uncommon $[11 \bullet \bullet, 31,33 \bullet \cdot$. A deranged coagulation profile is reported to occur in $20-25 \%$ of cases, although disseminated intravascular co-agulopathy (DIC) is usually confined to cases where there is severe liver dysfunction due to hepatic infiltration by blast cells [30-32, 33・•]. Hepatic dysfunction is manifested by severe conjugated hyperbilirubinaemia and often, but not always, accompanied by elevated transaminases $[30-32,33 \bullet \bullet, 39]$. The only one of these laboratory features that is specific for a diagnosis of TAM is a high number of circulating blast cells. However, one of the most challenging aspects of diagnosis of TAM has been establishing whether or not there is a threshold value for the percentage of blasts that is reliable for diagnosis in the absence of molecular confirmation by GATA1 mutation analysis.

Blast count, morphology and immunophenotype Although TAM is characterised by increased peripheral blood blasts, it is now known that blast cells are seen on the blood film of almost all neonates ( $98 \%$ ) with Down syndrome and may account for $15-20 \%$ of the circulating leucocytes in neonates shown to have no GATA1 mutations [11••]. There is no internationally agreed definition of a percentage blast threshold that constitutes 'increased peripheral blood blast cells'. In the Oxford Imperial Down Syndrome Cohort (OIDSC) Study, we addressed this question by prospectively classifying cases with blasts of $>10 \%$ and a GATA1 mutation in the first 14 days of life as TAM. In the preliminary analysis of the first 200 neonates with Down syndrome recruited into the study, $17(8.5 \%)$ fulfilled these criteria for a diagnosis and these criteria identified all neonates with clinical features of TAM, including all with severe disease $[11 \bullet \cdot]$. This analysis also showed that $25 \%$ of neonates with blasts $>10 \%$ do not have a GATA1 mutation even when very sensitive NGS-based methods are used. On the other hand, 18/70 neonates in the OIDSC study $(26 \%$ ) neonates with blasts $\leq 10 \%$ (range $1-10 \%$ ) had a GATA1 mutation when NGS-based methods were used; these cases had no clinical and haematological features suggestive of TAM and were designated 'silent TAM'. Taken together these data indicate that an accurate diagnosis of TAM relies on both the presence of blasts and a GATA1 mutation and suggest that a blast threshold of $>10 \%$ will identify all neonates with Down syndrome with TAM who may require chemotherapy and close monitoring during the neonatal period. However, this blast threshold is not specific for TAM and is not sufficiently sensitive to identify the majority of neonates who have GATA1 mutations. Typically, the blast cells in TAM are described as megakaryoblastic with cytoplasmic blebbing and basophilic cytoplasm; however, in our experience the morphology of the blasts is highly variable. Similarly, the immunophenotype of the blast cells is highly variable; the characteristic pattern of co-expression of stem cell markers (CD34 and CD117), myeloid markers (CD33/CD13), platelet glycoproteins (CD36, CD42, CD61) together with CD56 and CD7 is heterogeneous both within and between cases [35-38]. At present, there is no distinguishing morphological and immunophenotypic profile that can accurately discriminate TAM from cases where there are no GATA1 mutations [11••].

\section{Silent TAM}

As mentioned above, at least half of all Down syndrome neonates with GATA1 mutations have a peripheral blood blast percentage of $1-10 \%$ and have no clinical features associated 
Table 1 Clinical and haematological features of neonates with Down syndrome with and without GATA1 mutations

\begin{tabular}{llll}
\hline Clinical feature (\% neonates) & TAM $^{\mathrm{a}}$ & Silent TAM $^{\mathrm{b}}$ & Down syndrome (no GATA1 mutations) \\
\hline Hepatomegaly & 40 & $<5$ & 4 \\
Splenomegaly & 30 & $<1$ & $<1$ \\
Skin rash & 11 & $<1$ & $<1$ \\
Pericardial/pleural effusion & 9 & $<1$ & $<1$ \\
Jaundice & 70 & 60 & $50-60$ \\
Abnormal LFTs & 25 & $<10$ & $<10$ \\
Abnormal coagulation & $10-25$ & $\sim 5$ & $\sim 5$ \\
Thrombocytopenia $\left(<100 \times 10^{9} / 1\right)$ & 50 & 50 & 50 \\
Leucocytosis $\left(>26 \times 10^{9} / 1\right)$ & $\sim 50$ & 10 & $10-15$ \\
Anaemia $(<130 \mathrm{~g} / \mathrm{L})$ & $5-10$ & $<5$ & $1-5$ \\
\hline
\end{tabular}

Data based on information from refs. [11••, 30-32, 33••]

${ }^{\text {a }}$ Peripheral blood blasts $>10 \%$ and one or more acquired GATA1 mutations

${ }^{\mathrm{b}}$ Silent TAM: Peripheral blood blasts $\leq 10 \%$ and one or more acquired GATA1 mutations with TAM (Table 1) [11••]. This is an important group of neonates because the presence of the GATA1 mutation means that they are at risk of subsequently developing ML-DS if the mutant GATA1 clone persists [11••]. It is likely that the reason for the lack of clinical features is the small size of the mutant GATA1 clone at birth since the OIDSC study showed a strong correlation between the size of the mutant clone and the percentage of peripheral blood blasts $[11 \bullet \cdot]$.

\section{TAM and Silent TAM: GATA1 Mutation Analysis}

The recognition of silent TAM means that detection of GATA1 mutations for clinical diagnosis requires sensitive as well as specific and reliable methods. Current available methodologies for GATA1 mutation analysis are direct Sanger sequencing (sensitivity 10-30\%), dHPLC (sensitivity 2-10\%) and various methods of NGS (sensitivity $0.3-2 \%$ ) [9, 10, 11••, 12••]. Each method has technical limitations, advantages and disadvantages, but only NGS-based methods are sufficiently sensitive for initial diagnosis as neither direct Sanger sequencing nor dHPLC are able to reliably detect small GATA1 mutant clones $(<10 \%)$ that are of clinical significance [11••]. The value of monitoring mutant GATAl clones after diagnosis is currently not clear; this would require an extremely sensitive method and an important limitation is that neonates with TAM may have more than one GATA1 mutant clone and that ML-DS may develop from either or both major and minor GATA1 clones present at birth $[11 \bullet \cdot, 12 \bullet \cdot]$.

\section{Natural History of TAM and Progression to ML-DS}

Most neonates with TAM ( $>80 \%)$ undergo spontaneous resolution of both clinical and laboratory abnormalities within 3 months after birth with a 5-year overall survival of $80 \%$ and event-free survival of $\sim 60 \%$ [30-32, 33••]. Complete remission is often characterised first by normalisation of blood counts and disappearance of peripheral blasts followed by resolution of clinical symptoms such as hepatomegaly [39]. The overall mortality is reported to be $\sim 20 \%$, however, only half of the deaths are directly attributable to TAM usually due to hepatic failure secondary to fibrosis and blast cell infiltration $[30-32,33 \cdot \bullet]$.

Estimates of the risk of progression of TAM to ML-DS are mainly based on retrospective studies and suggest that 20 $30 \%$ of neonates with TAM will subsequently present with ML-DS $[30-32,33 \bullet \cdot$. Since it is now known that the frequency of GATA1 mutations at birth is much higher than previously realised (25-30\% of all neonates with Down syndrome) and since population-based estimates of the frequency of MLDS indicate that $\sim 1.5 \%$ of children with Down syndrome will develop this leukaemia before the age of 5 years [1], this suggests that the risk of progression is lower than these original estimates (around 5-10\%) given that silent TAM also has the potential to transform to ML-DS. In some cases, there is overt progression/evolution of TAM to ML-DS with persistent abnormal haematology and an indolent myelodysplastic syndrome; in other cases, there is a variable apparent remission before development of ML-DS [11••, 30, 31]. GATA1 mutations are detected in all cases of ML-DS $[11 \bullet, 12 \bullet \cdot]$ and are therefore essential for progression to ML-DS. Factors, which reliably predict transformation of TAM to ML-DS, have not been identified yet. The type of GATA1 mutation does not seem to play a role [10]. Data on the size of the GATA1 mutant clone at birth as a predictor of later ML-DS are too preliminary at present. The only clinical factor shown in multivariate analysis to predict transformation of TAM to ML-DS is the presence of pleural effusion in the neonatal period [31].

\section{TAM: Management}

Most neonates with TAM undergo spontaneous resolution and do not need treatment. However, neonates with progressive 
Table 2 Mortality and transformation to myeloid leukaemia of Down syndrome (ML-DS) in neonates with Down syndrome and transient abnormal myelopoiesis (TAM)*

\begin{tabular}{llllll}
\hline No. of patients & Massey & Klusmann & Muramatsu \\
{$[30]$} & {$[31]$} & $\begin{array}{l}\text { Gamis } \\
{[32]}\end{array}$ & Total \\
& 47 & 146 & 70 & 135 & 398 \\
\hline Early death & $8(17 \%)$ & $22(15 \%)$ & $16(23 \%)$ & $29(21 \%)$ & $75(19 \%)$ \\
TAM-associated hepatic & $8(17 \%)$ & $7(5 \%)$ & $11(16 \%)$ & $13(10 \%)$ & $39(10 \%)$ \\
$\quad$ failure & 0 & $6(4 \%)$ & $4(5.7 \%)$ & $1(0.7 \%)$ & $11(3 \%)$ \\
Other TAM deaths & 0 & $9(6 \%)$ & $1(1.4 \%)$ & $15(11 \%)$ & $25(6 \%)$ \\
Non-TAM deaths & $9(23 \%)$ & $29(23 \%)$ & $12(22 \%)$ & $21(20 \%)$ & $71(22 \%)$ \\
ML-DS (of survivors) &
\end{tabular}

* Clinically diagnosed TAM. Data based on information from refs. [30-32, 33••] life-threatening symptoms such as hydrops fetalis, extreme leucocytosis (WBC >100 $\times 10^{9} / 1$ ), hepatopathy, DIC with bleeding, renal and/or cardiac failure may benefit from chemotherapy as the mortality rate may be up to $20 \%$ [30-32, $33 \bullet \bullet, 39]$. A summary of the outcome of treatment from these studies is shown in Table 2. As TAM blasts appear to be very sensitive to cytarabine [40] and early observational studies showed promising results with very low doses of cytarabine [41], currently used regimens are based on this approach. The Berlin-Frankfurt-Münster group recommended treatment with cytarabine (0.5-1.5 mg/kg for 3-12 days) for neonates with TAM and clinical impairment due to thrombocytopenia, signs of cholestasis or liver dysfunction or high white cell count $\left(>50 \times 10^{9} / 1\right)$ [31]. Out of 146 patients, 28 received treatment with cytarabine many of which had hepatic fibrosis and required intensive support. Survival in the treated and untreated groups was very similar (5-year overall survival $78 \pm 8$ vs. 85 $\pm 3 \%, p=0.44$ ), suggesting that treatment might have been beneficial given that the treated neonates had much more severe disease [31]. The Children's Oncology Group identified 38 of 135 patients as having life-threatening symptoms and 24 received cytarabine, given as a continuous infusion at a dose of $3.33 \mathrm{mg} \mathrm{kg}^{-} / \mathrm{day}^{-1}$ for 7 days. The survival rate for the treatment group was disappointing (51\%) most likely reflecting both the severity of the disease and the high rate of haematological toxicity ( $96 \%$ grade $3 / 4$ myelosuppression) perhaps because of the higher dose and continuous infusion regimen [33••]. More recently, a preliminary report from Muramatsu et al. of a large study in neonates with TAM reported a significant improvement in 1 year survival when neonates with extreme leucocytosis $\left(>100 \times 10^{9} / 1\right)$ were treated with cytarabine [52]. However, there is no evidence at present that treatment with cytarabine has a significant impact on the likelihood of disease progression to ML-DS [31, 33••].

\section{ML-DS: Clinical Features}

ML-DS is classified as a specific subtype of AML in the World Health Organisation (WHO) classification [42]. This leukaemia is unique to Down syndrome and has several distinct features. Firstly, ML-DS presents at a median age of 1-
1.8 years and is rare after the age of 4 years $[43,44]$. Secondly, most cases of ML-DS have a clinical history consistent with preceding TAM in the neonatal period, and for those that have no such history, the most likely reason is the absence of appropriate diagnostic tests at birth [11••]. Consistent with this, GATA1 mutations are found on neonatal bloodspots from neonates with ML-DS even in the absence of an antecedent history of TAM [9]. ML-DS often shows an indolent presentation with myelodysplasia and progressive pancytopenia, in particular thrombocytopenia and leucopenia, with a low percentage of circulating blasts for many months before the development of ML-DS [43, 45, 46]. Since the circulating blast count is often low in ML-DS and the predominant haematological picture may be of slowly progressive pancytopenia, a bone marrow aspirate is usually essential for the diagnosis of ML-DS. However, this is often associated with a 'dry tap' secondary to marked bone marrow fibrosis and a bone marrow trephine may be necessary to confirm ML-DS - it is not clear that the conventional bone marrow blast threshold used in acute myeloid leukaemia is of value in ML-DS in view of the natural history of the condition and the difficulty in obtaining a representative sample.

\section{ML-DS: Laboratory Features}

Almost all patients with ML-DS have thrombocytopenia and most also have anaemia and neutropenia. In contrast to TAM, the leucocyte count is usually low. However, the blast cells are similar to those in TAM, with a typical megakaryoblastic morphology [43, 47] and co-expression of stem/progenitor cell markers (CD34, CD117), myeloid (CD33), megakaryocytic (CD42b and CD41) and erythroid markers (CD36 and glycophorin A) as well as CD7 [35, 37, 47]. ML-DS has a distinct cytogenetic profile compared with sporadic AML in children without Down syndrome in that the favourable cytogenetic changes such as $A M L-E T O \mathrm{t}(8 ; 21), P M L-R A R A$ $\mathrm{t}(15 ; 17), M L L \mathrm{t}(9 ; 11)$ and $C B F B$ MYH11 inv[16] nor the acute megakaryoblastic leukaemia-associated translocations RBM15-MKL1 $\mathrm{t}(1 ; 22)$ and $\mathrm{t}(1 ; 3)$ occur in ML-DS [48, 49]. Instead, several karyotypic abnormalities are more frequent in ML-DS than in children without Down syndrome, including 
trisomy 8 , trisomy 11 , trisomy $21, \operatorname{del}(6 q), \operatorname{del}(7 p), \operatorname{del}(16 q)$ and $\operatorname{dup}(1 \mathrm{p})$ [49]. GATA1 mutations are always present in ML-DS blasts and, where available, targeted or genomewide next-generation sequencing usually reveals mutations in additional known oncogenes, such as the cohesin genes, as described above $[12 \bullet \bullet, 13]$.

\section{ML-DS: Treatment and Outcome}

A number of studies show that children with ML-DS have better outcomes compared with children without Down syndrome with long-term survival of 74-91 \% (Nordic group 10year OS $74 \%, n=61$; the Children's Cancer Group (CCG) 5year OS $79 \%, n=161$; BFM study group 3-year OS $91 \%$, $n=67$ and the Medical Research Council 5-year OS, $75 \%$, $n=36$ ) $[33 \cdot \bullet, 43,45,50]$. Early studies showed that treatment failure in children with Down syndrome due mainly to chemotherapy-related toxicity, particularly related to anthracylines, with a higher rate of induction deaths in MLDS [45]. Most groups now use a reduced dose of anthracycline that is less toxic and appears to give similar efficacy [55-57]. The outlook for children with ML-DS who relapse is very poor. In a review of the Japanese data, Taga et al. reported a 3 -year overall survival rate of $25.9 \%$ [51], similar to the $21 \%$ overall survival in the POG 9421 and CCG-22891 AML studies [52]. There appears to be no role for allogeneic stem cell transplant (SCT) in first-line therapy for ML-DS due to efficacy of chemotherapy and the ongoing high rate of toxicity associated with SCT. Even the role of SCT as salvage therapy is unclear. A recent review by the Center for International Blood and Marrow Transplant Research (CIBMTR) of outcome data from 28 children transplanted for ML-DS compared with 80 non-Down syndrome children with AML reported a 3year probability of overall survival of only $19 \%$ due to the high rate of relapse and transplant-related mortality [53]. The results of a small retrospective study in 15 patients suggest that transplant-related toxicity might be reduced by using reduced intensity conditioning regimens $(n=5 ; 80 \%$ event-free survival) compared with standard myeloabalative regimens $(n=$ $10 ; 10 \%$ event-free survival) [54]; however, these results remain to be confirmed in larger studies.

\section{Conclusion}

Children with Down syndrome have a markedly increased risk ( 150-fold) of developing acute myeloid leukaemia, known as ML-DS) compared with children without Down syndrome. ML-DS is preceded by a clonal neonatal preleukaemic disorder, known as TAM, which maybe clinically overt or silent. TAM and ML-DS have unique biological, cytogenetic and molecular characteristics. There are at least three distinct steps in the pathogenesis of ML-DS. First, trisomy 21 perturbs fetal haematopoiesis, providing the ideal cellular context for the second step: transformation of these fetal haematopoietic cells by acquired N-terminal truncating mutations in the GATA1 gene to produce the clinical syndrome TAM. While the majority of cases of TAM resolve without sequlae as the GATA1 mutation is lost, $\sim 10 \%$ of children harbour residual GATA1-mutant cells which then, in the third step, acquire transforming mutations in additional oncogenes leading to ML-DS. Uncovering the mechanisms which underlie these events remains an exciting challenge and is at last beginning to offer real prospects of translation of these finding into useful therapeutic advances for children with Down syndrome so that we can improve treatment and outcome by investigating new agents that could potentially improve their leukaemia-free survival without additional toxicity.

\section{Compliance with Ethical Standards}

Conflict of Interests Neha Bhatnagar, Laure Nizery, Oliver Tunstall, Paresh Vyas and Irene Roberts each declare no potential conflicts of interest.

Human and Animal Rights and Informed Consent This article does not contain any studies with human or animal subjects performed by any of the authors.

Open Access This article is distributed under the terms of the Creative Commons Attribution 4.0 International License (http:// creativecommons.org/licenses/by/4.0/), which permits unrestricted use, distribution, and reproduction in any medium, provided you give appropriate credit to the original author(s) and the source, provide a link to the Creative Commons license, and indicate if changes were made.

\section{References}

Papers of particular interest, published recently, have been highlighted as:

- Of importance

•• Of major importance

1. Hasle H, Clemmensen IH, Mikkelsen M. Risks of leukaemia and solid tumours in individuals with Down's syndrome. Lancet. 2000;355:165-9.

2. Patja K, Pukkala E, Sund R, Iivanainen M, Kaski M. Cancer incidence of persons with Down syndrome in Finland: a population-based study. Int J Cancer. 2006;118:1769-72.

3. Malinge S, Izraeli S, Crispino JD. Insights into the manifestations, outcomes, and mechanisms of leukemogenesis in Down syndrome. Blood. 2009;113:2619-28.

4. Roberts I, Izraeli S. Haematopoietic development and leukaemia in Down syndrome. Br J Haematol Epub Aug. 2014;22:2014.

5. Wechsler J, Greene M, McDevitt M, Anastasi J, Karp J, Le Beau M, et al. Acquired mutations in GATAl in the megakaryoblastic leukemia of Down syndrome. Nat Genet. 2002;32:148-52.

6. Rainis L, Bercovich D, Strehl S, Teigler-Schlegel A, Stark B, Trka J, et al. Mutations in exon 2 of GATAl are early events in megakaryocytic malignancies associated with trisomy 21. Blood. 2003;102:981-6. 
7. Hitzler JK, Cheung J, Li Y, Scherer SW, Zipursky A. GATA1 mutations in transient leukemia and acute megakaryoblastic leukemia of Down syndrome. Blood. 2003;101:4301-4.

8. Groet J, McElwaine S, Spinelli M, Rinaldi A, Burtscher I, Mulligan C, et al. Acquired mutations in GATA1 in neonates with Down's syndrome with transient myeloid disorder. Lancet. 2003;361:1617-20.

9. Ahmed M, Sternberg A, Hall G, Thomas A, Smith O, O'Marcaigh A, et al. Natural history of GATA1 mutations in Down syndrome. Blood. 2004;103:2480-9.

10. Alford K, Reinhardt K, Garnett C, Norton A, Bohmer K, von Neuhoff C, et al. Blood. 2011;118:2222-38.

11.• Roberts I, Alford K, Hall G, Juban G, Richmond H, Norton A, et al. GATA1-mutant clones are frequent and often unsuspected in babies with Down syndrome: identification of a population at risk of leukemia. Blood. 2013;122:3908-17. This paper reports interim analysis from the prospective multi-centre Oxford Imperial Down syndrome cohort study which showed the high frequency of acquired GATA1 mutations in neonates with Down syndrome and described Silent TAM for the first time.

12.• Yoshida K, Toki T, Okuno Y, Kanezaki R, Shiraishi Y, Sato-Otsubo A, et al. The landscape of somatic mutations in Down syndrome-related myeloid disorders. Nat Genet. 2013;45:1293-9. This paper provides the first comprehensive analysis of the molecular landscapte of ML-DS and TAM.

13. Nikolaev S, Santoni F, Vannier A, Falconnet E, Giarin E, Basso G, et al. Exome sequencing identifies putative drivers of progression of transient myeloproliferative disorder to AMKL in infants with Down syndrome. Blood. 2013;122:554-61

14. Tunstall-Pedoe O, Roy A, Karadimitris A, de la Fuente J, Fisk N, Bennett P, et al. Abnormalities in the myeloid progenitor compartment in Down syndrome fetal liver precede acquisition of GATA1 mutations. Blood. 2008;112:4507-11.

15.• Roy A, Cowan G, Mead AJ, Filippi S, Bohn G, Chaidos A, et al. Perturbation of fetal liver hematopoietic stem and progenitor cell development by trisomy 21. Proc Natl Acad Sci U S A. 2012;109: 17579-84. This paper provides the first description of global perturbation of fetal haematopoiesis by trisomy 21 at haematopoietic stem and progenitor cell level.

16. Kirsammer G, Jilani S, Liu H, Davis E, Gurbuxani S, Le Beau MM, et al. Highly penetrant myeloproliferative disease in the Ts65Dn mouse model of Down syndrome. Blood. 2008;111:767-75.

17. Carmichael CL, Majewski IJ, Alexander WS, Metcalf D, Hilton DJ, Hewitt CA, et al. Hematopoietic defects in the Ts1Cje mouse model of Down syndrome. Blood. 2009;113:1929-37.

18. Alford KA, Slender A, Vanes L, Li Z, Fisher EM, Nizetic D, et al. Perturbed hematopoiesis in the Tc1 mouse model of Down syndrome. Blood. 2010;115:2928-37.

19. Malinge S, Bliss-Moreau M, Kirsammer G, Diebold L, Chlon T, Gurbuxani S, et al. Increased dosage of the chromosome 21 ortholog Dyrk1a promotes megakaryoblastic leukemia in a murine model of Down syndrome. J Clin Invest. 2012;122:948-62.

20. Birger Y, Goldberg L, Chlon T, Goldenson B, Muler I, Schiby I, et al. Perturbation of fetal hematopoiesis in a mouse model of Down syndrome's transient myeloproliferative disorder. Blood. 2013;122:988-98.

21. Chou S, Byrska-Bishop M, Tober J, Yao Y, VanDorn D, Opalinska J, et al. Trisomy 21-associated defects in human primitive hematopoiesis revealed through induced pluripotent stem cells. Proc Natl Acad Sci U S A. 2012;109:17573-8.

22. MacLean G, Menne T, Guo G, Sanchez D, Park I, Daley G, et al. Altered hematopoiesis in trisomy 21 as revealed through in vitro differentiation of isogenic human pluripotent cells. Proc Natl Acad Sci U S A. 2012;109:17567-72.

23. Liu B, Filippi S, Roy A, Roberts I. Stem and progenitor cell dysfunction in human trisomies. EMBO Rep. 2015;16:44-62.
24. Muntean A, Ge Y, Taub J, Crispino J. Transcription factor GATA-1 and Down syndrome leukemogenesis. Leukemia \& lymphoma. 2006;47: 986-97.

25. Salek-Ardakani S, Smooha G, de Boer J, Seibre N, Morrow M, Rainis L, et al. ERG is a megakaryocytic oncogene. Cancer Res. 2009;69: 4665-73.

26. Toki T, Kanezaki R, Kobayashi E, Kaneko H, Suzuki M, Wang R, et al. Naturally occurring oncogenic GATA1 mutants with internal deletions in transient abnormal myelopoiesis in Down syndrome. Blood. 2013;121:3181-4

27. Banno K, Omori S, Hirata N, Nakagawa K, Nishimura M, Ohtaka M, et al. Systematic cellular disease models reveal synergistic interaction of trisomy 21 and GATA1 mutations in hematopoietic abnormalities. Cell Rep. 2016;15:1228-41.

28. Caye A, Strullu M, Guidez F, Cassinat B, Gazal S, Fenneteau O, et al. Juvenile myelomonocytic leukaemia displays mutations in components of the RAS pathway and the PRC2 network. Nat Genet. 2015;47:1334-40.

29. Klusmann J-H, Godinho F, Heitmann K, Maroz A, Koch M, Reinhardt D, et al. Developmental stage-specific interplay of GATAI and IGF signaling in fetal megakaryopoiesis and leukemogenesis. Genes Dev. 2010;24:1659-72.

30. Massey GV, Zipursky A, Chang M, Doyle J, et al. A prospective study of the natural history of transient leukemia in neonates with Down syndrome: Children's Oncology Group study POG-9481. Blood. 2006;107:4606-13.

31. Klusmann J-H, Creutzig U, Zimmermann M, Dworzak M, et al. Treatment and prognostic impact of transient leukemia in neonates with Down syndrome. Blood. 2008;111:2991-8.

32. Muramatsu H, Kato K, Watanabe N, Matsumoto K, et al. Risk factors for early death in neonates with Down syndrome and transient leukaemia. Br J Haematol. 2008;142:610-5.

33.• Gamis AS, Alonzo T, Gerbing R, Hilden J, et al. Natural history of transient myeloproliferative disorder clinically diagnosed in Down syndrome neonates: a report from the Children's Oncology Group Study A2971. Blood. 2011;118:6752-9. This paper describes a large prospective study (COG Study A2971) of clinically-diagnosed TAM with a particular emphasis on the frequency and significance of life-thtreatening symptoms.

34. Tamblyn J, Norton A, Spurgeon L, Donovan V, Bedford Russell A, Bonnici J, et al. Prenatal therapy in transient abnormal myelopoiesis: a systematic review. Arch Dis Child Fetal Neonatal Ed. 2016;101:67-71. This paper is the first comprehensive review of the clinical features, haematological features and outcome of TAM presenting in utero.

35. Karandikar NJ, Aquino DB, McKenna RW, Kroft SH. Transient myeloproliferative disorder and acute myeloid leukemia in Down syndrome. An immunophenotypic analysis. Am J Clin Pathol. 2001;116: 204-10.

36. Giordon F, Favre B, Couillaud G, et al. Immunophenotype of a transient myeloproliferative disorder in a newborn with trisomy 21 . Cytometry. 2000;42:118-22.

37. Langerbrake C, Creutzig U, Reinhardt D. Immunophenotype of Down syndrome acute myeloid leukemia and transient myeloproliferative disease differs significantly from other diseases with morphologically identical or similar blasts. Klin Padiatr. 2005;217:126-34.

38. Boztug H, Schumich A, Potschger U, Muhlegger N, Kolenova A, Reinhardt K, et al. Blast cell deficiency of CD11a as a marker of acute megakaryoblastic leukaemia and transient myeloproliferative disease in children with and without Down syndrome. Cytometry B Clin Cytom. 2013;84:370-8.

39. Park M, Sotomatsu M, Ohki K, Arai K, Maruyama K, Kobayashi T, et al. Liver disease is frequently observed in Down syndrome patients with transient abnormal myelopoiesis. Int J Hematol. 2014;99:154-61.

40. Zwaan C, Kaspers G, Pieters R, Hahlen K, Janka-Schaub G, van Zantwijk C, et al. Different drug sensitivity profiles of acute myeloid and lymphoblastic leukemia and normal peripheral blood 
mononuclear cells in children with and without Down syndrome. Blood. 2002;99:245-51.

41. Al-Kasim F, Doyle J, Massey G, Weinstein H, Zipursky A. Incidence and treatment of potentially lethal diseases in transient leukemia of Down syndrome: Pediatric Oncology Group Study. J Pediatr Hematol Oncol. 2002;24:9-13.

42. Hasle H, Niemeyer CM, Chessells JM, Baumann I, et al. A pediatric approach to the WHO classification of myelodysplastic and myeloproliferative diseases. Leukemia. 2003;17:277-82.

43. Creutzig U, Zimmermann M, Ritter J, Reinhardt D, Hermann J, Henze $\mathrm{G}$, et al. Treatment strategies and long-term results in paediatric patients treated in four consecutive AML-BFM trials. Leukaemia. 2005; 19:2030-42.

44. Hasle H, Abrahamsson J, Arola M, Karow A, et al. Myeloid leukemia in children 4 years or older with Down syndrome often lacks GATA1 mutation and cytogenetics and risk of relapse are more akin to sporadic AML. Leukemia. 2008;22:1428-30.

45. Rao A, Hills R, Stiller C, Gibson B, et al. Treatment for myeloid leukaemia of Down syndrome: population-based experience in the UK and results from the Medical Research Council AML 10 and AML 12 trials. Br J Haematol. 2006;132:576-83.

46. Webb D, Roberts I, Vyas P. Haematology of Down syndrome. Arch Dis Child Fetal Neonatal Ed. 2007;92:503-7.

47. Yumura-Yagi K, Hara J, Tawa A, Kawa-Ha K. Phenotypic characteristics of acute megakaryocytic leukemia and transient abnormal myelopoiesis. Leuk Lymphoma. 1994;13:393-400.

48. Kurkijan C, Patel S, Kamble R, Dunn S, Kern W, Kharfan-Dabaja M. Acute promyelocytic leukemia and constitutional trisomy 21. Cancer Genet Cytogenet. 2006;165:176-9.

49. Forestier E, Izraeli S, Beverloo B, Haas O, Pession A, Michalova K, et al. Cytogenetic features of acute lymphoblastic and myeloid leukemias in pediatric patients with Down syndrome: an iBFM-SG study. Blood. 2008;111:1575-83.
50. Ravindranath Y. Down syndrome and acute myeloid leukemia: The paradox of increased risk for leukemia and heightened sensitivity to chemotherapy. J Clin Oncol. 2003;21:3385-7.

51. Taga T, Saito A, Kudo K, Tomizawa D, Terui K, Moritake H, et al. Clinical characteristics and outcome of refractory/relapsed myeloid leukaemia in children with Down syndrome. Blood. 2012;120:1810-5.

52. Muramatsu H, Watanabe T, Hasegawa D, et al. Prospective Study of 168 Infants with Transient Abnormal Myelopoiesis with Down Syndrome: Japan Pediatric Leukemia/Lymphoma Study Group, TAM-10 Study (Abstract 1311; 57th Annual Meeting of the American Society of Hematology). 2015.

53. Hitzler J, He W, Doyle J, Cairo M, Camitta B, Chan KW, et al. Outcome of transplantation for acute myelogenous leukaemia in children with Down syndrome. Biology of Blood and Marrow Transplant. 2013;19:893-7.

54. Muramatsu H, Sakaguchi H, Taga T, Tabuchi K, Adachi S, Inoue M, et al. Reduced intensity conditioning in allogeneic stem cell transplantation for AML with Down syndrome. Pediatric Blood Cancer. 2014;61:925-7.

55. Taga T, Watanabe T, Tomizawa D, Kudo K, Terui K, Moritake H, et al. Preserved high probability of overall survival with significant reduction of chemotherapy for myeloid leukaemia in Down syndrome: a nationwide prospective study in Japan. Pediatric Blood and Cancer. 2016;63:248-54.

56. Creutzig U, Reinhardt D, Diekamp S, Dworzak M, Stary J, Zimmermann M. AML patients with Down syndrome have a high cure rate with AML-BFM therapy with reduced intensity. Leukemia. 2005; 19:1355-60.

57. Sorrell A, Alonzo T, Hilden J, Gerbing R, Loew T, Hathaway L, et al. Favorable survival maintained in children who have myeloid leukemia associated with Down syndrome using reduced-dose chemotherapy on Children's Oncology Group Trial A2971. Cancer. 2012;118:4806-14. 